\title{
Development and Evaluation of an Electron Beam Source for Microscopy and Its Applications
}

\author{
Seungjoon Ahn*, Tae-Sik Oh, and Ho Seob Kim \\ Department of Information Display, Sun Moon University, Tangjeong-myeon, Asan 336-708, Korea \\ Seong Joon Ahn \\ Department of I\&C Engineering, Sun Moon University, Tangjeong-myeon, Asan 336-708, Korea
}

(Received January 29, 2010 : revised March 30, 2010 : accepted March 30, 2010)

\begin{abstract}
We have developed an efficient electron beam (e-beam) source, a microcolumn, that can be used as a source module for of microscopy and its applications. To obtain a low operating voltage, a very sharp cold field electron emitter was developed by electrochemically etching a tungsten wire. Laser diffraction was used for the fabrication of high-quality electron lenses and for their precise alignment. The measurement of the e-beam currents, and SEM images captured by the microcolumn confirmed the potential of the device as a very good e-beam source.
\end{abstract}

Keywords: Electron beam source, THz, Microcolumn, Microscopy, Diffraction pattern

OCIS codes : (120.0120) Instrumentation, measurement, and metrology; (040.2235) Far infrared or terahertz; (180.5810) Scanning microscopy; (220.4000) Microstructure fabrication

\section{INTRODUCTION}

Mankind has utilized electromagnetic (EM) waves, including optical waves, throughout history. Each region of their wide spectrum has its own applications - optical and wireless communications, lighting, optical lithography[1, 2], radio and television broadcasting, sterilization of medical equipment, detection of many physical quantities, diagnosis of problems of the human body, to name a few. Most of today's advanced technologies employ EM waves. For each application of EM waves, appropriate wave sources have been developed. For example, modern optical networks having huge transmission capacity could not have been realized if it had not been for compact infrared semiconductor lasers [3].

Recently there has been much interest in the $\mathrm{THz}$ region[4-6] a relatively unexplored region of EM waves. This region is located between 0.3 and $10 \mathrm{THz}$ in frequency, or equivalently, between 30 and $1000 \mu \mathrm{m}$ in wavelength. Despite great applicability to examining living tissues like human cells, there is no $\mathrm{THz}$ source which is compact and easy to use.
As an effort to resolve such a problem, in this work, we have developed a microcolumn that can be used as a convenient source module. There has been steady research in the microcolumns that can be used for microscopy and its applications. While the principle of the microcolumn is the same as that of conventional e-beam equipment, its structure can be simplified very much compared to that of the convential e-beam equipment due to the miniaturized electron lenses. The characteristics of microcolumns have shown the potential of microcolumns as widely tunable $\mathrm{THz}$ sources (including far infrared).

\section{DEVELOPMENT OF THE MICROCOLUMN}

The microcolumn is composed of several elements including an electron emitter, a scan deflector, and electron lenses. Among these elements, the electron emitter is the source of the electron beam (e-beam), and its characteristics determine the whole performance of the system. In this work, we have fabricated a cold field emitter (CFE) having a very sharp tip by electro-chemically etching tungsten (W)[7]. In

\footnotetext{
*Corresponding author: sjan@sunmoon.ac.kr

Color versions of one or more of the figures in this paper are available online.
} 
the conventional CFE that has been used in large-scale electron columns, the diameter of the tip is larger than 100 $\mathrm{nm}$. With such a large radius of curvature, the tip cannot emit electrons unless the voltage bias applied to the tip is sufficiently high (typically higher than $10 \mathrm{kV}$ ).

Since such a high voltage causes physical damage to the micro-lenses of the microcolumn, we have developed a very sharp tip with diameter much less than $50 \mathrm{~nm}$. Fig. 1 shows the scanning electron microscope (SEM) image of the tip we have developed. With this sharp tip, we could decrease the voltage bias to as low as $-230 \mathrm{~V}$.

The resolution of the microcolumn system is significantly influenced by aberrations such as astigmatism, coma, spherical aberration and chromatic aberration, which are, in turn, dependent on the eccentricity of the lens aperture and alignment of the system components. Two sets of lenses are required in the microcolumn. One is a source lens set that extracts the electrons from the emitter and controls the diameter of the e-beam. The other is an Einzel lens set which concentrates the e-beam around the target point of the sample that is being processed by the microcolumn system $[8,9]$. Both source and Einzel lens sets were composed of three electron lenses in our microcolumns.
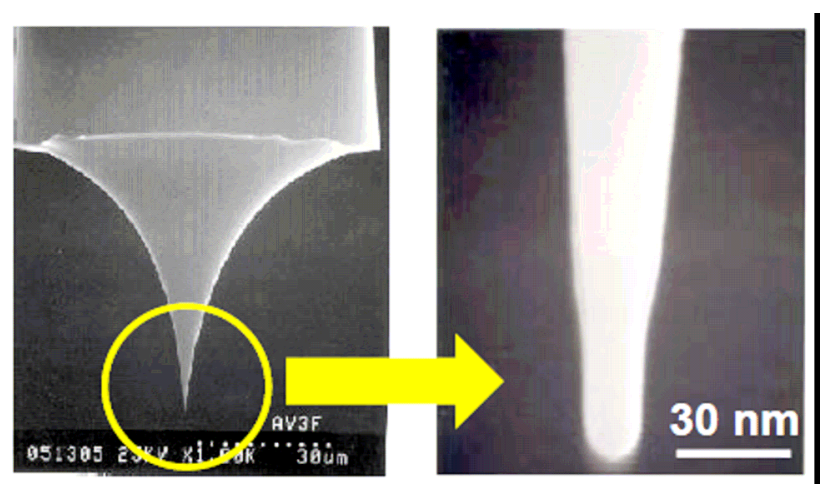

FIG. 1. The SEM image of the sharp tip fabricated by electro-chemical etching.
Each electron lens was fabricated by making a circular aperture in a $2-\mu \mathrm{m}$ thick $\mathrm{Si}$ membrane through semiconductor processing technologies like lithography, cleaning, and dry etching. The size of the membrane was $2 \times 2 \mathrm{~mm}^{2}$, and it was located at the center of a silicon chip whose dimension was $10 \times 10 \mathrm{~mm}^{2}$. Fig. 2 is an SEM image of the electron lens. The diameter of the lens aperture was $200 \mu \mathrm{m}$.

To investigate the shape of the lens aperture and the alignment of the lens assembly, we have used laser diffraction[10]. By illuminating each lens or lens assembly with a He-Ne laser, we have obtained the corresponding Fraunhofer diffraction pattern. When the aperture is a perfect circular disk, the intensity profile of the diffraction pattern follows the first-order Bessel function. Hence, by observing the diffraction pattern, we could select the electronic lenses having circular apertures.

We could also find the diameter of the aperture by combining the pattern data with the optical wavelength and the distance between the aperture and the diffraction pattern. This is a very precise and convenient method for measuring the size of the small circular hole. The lens assembly composed of several electronic lenses also shows the same diffraction pattern when they are in good alignment. We used this to adjust the position of the lenses in fabricating the microcolumn.

Fig. 3 shows (a) the diffraction pattern from two aligned Einzel lenses having $200-\mu \mathrm{m}$ apertures and (b) top-view microscope image of the assembled lens set. From the circular diffraction patterns and the photograph, we could confirm that the eccentricity of the apertures was almost zero and the alignment of the lenses was very good.

\section{CHARACTERISTICS OF THE MICROCOLUMN}

Fig. 4 is the experimental set-up for characterization of the microcolumn that we developed. In the figure, the set
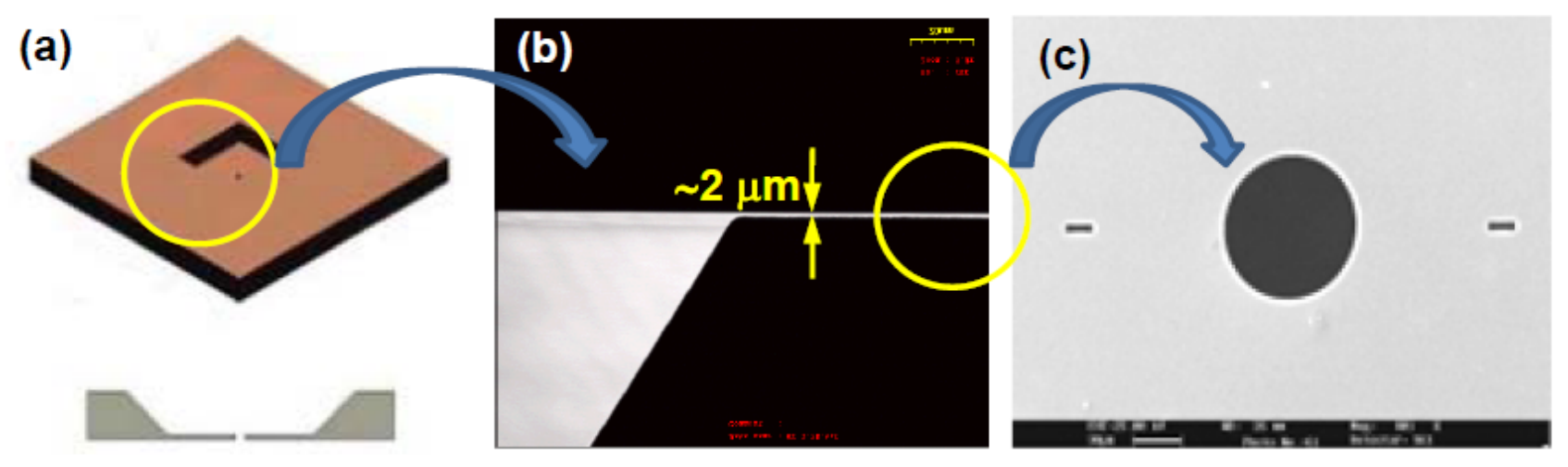

FIG. 2. (a) Electron lens fabricated by semiconductor processing. (b) A cross-sectional view of the electron lens. (c) SEM image of the electron lens having a diameter of $200 \mu \mathrm{m}$. 


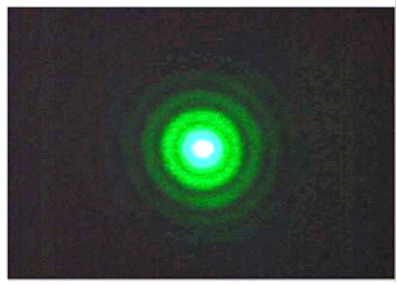

(a)

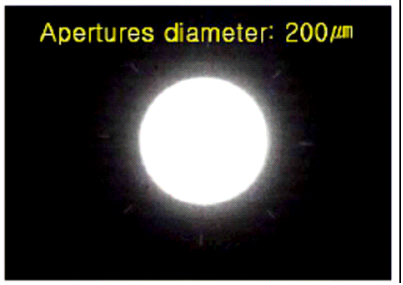

(b)

FIG. 3. (a) The diffraction pattern from two aligned Einzel lenses having $200-\mu \mathrm{m}$ apertures and (b) top-view microscope image of the assembled lens set.

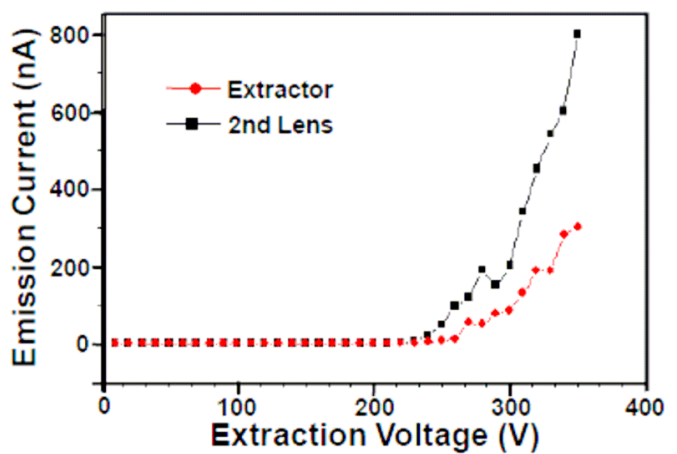

(a)

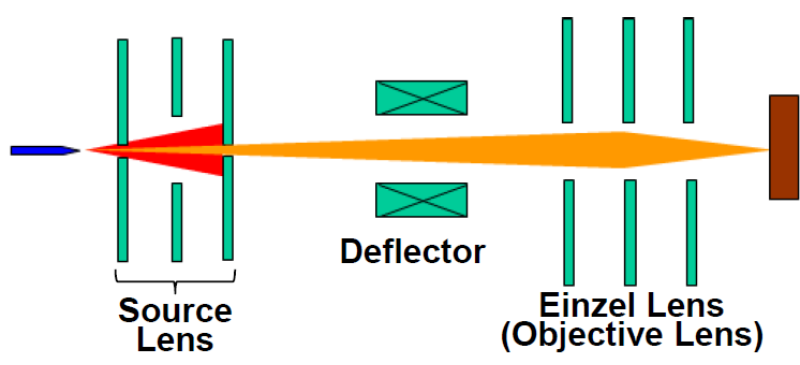

FIG. 4. Experimental set-up for characterization of the microcolumn.

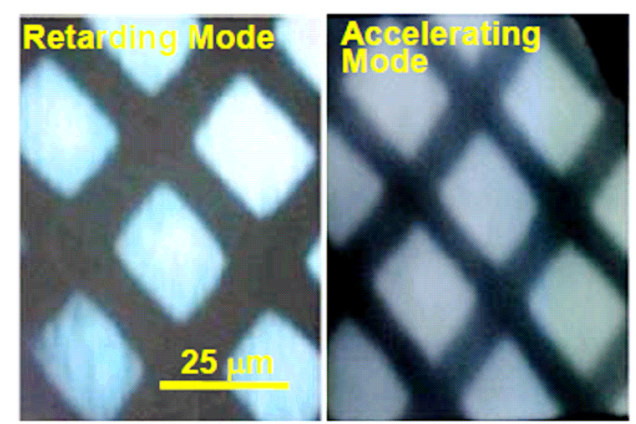

(b)

FIG. 5. (a) I-V curve of the microcolumn . (b) The image of $\mathrm{Cu}$ grid sample(mesh 1000) obtained by the microcolumn operating at retarding mode and accelerating mode.

of extractor, accelerator, and limiting aperture is called the source lens. Usually a negative bias is applied to the electron emitter and the source lens is grounded to extract the electrons from the sharp emitter tip. We have analyzed the characteristics of the microcolumn developed by measuring the e-beam currents at the first electron lens and at the sample with respect to the various bias voltages.

The electron emitter was characterized at the UHV pressure of $5 \times 10^{-10}$ torr. To accurately measure the e-beam current, we used a precision DC power supply that can provide $1-\mathrm{kV}$ DC voltage while maintaining peak-to-peak fluctuation less than $10 \mathrm{mV}$. The e-beam currents were measured using a pico-ammeter at the first aperture (extractor), at the second aperture, and at the sample. We also observed the images of $\mathrm{Cu}$ grid sample(mesh 1000) by using the microcolumn as the electron microscope.

Fig. 5(a) shows the measured e-beam currents. The electron emission began at bias voltage as low as $-230 \mathrm{~V}$, which was a significant reduction compared to the $-10 \mathrm{kV}$ bias of the conventional electron column, proving that the diameter of the emitter tip was much less than that of the typical emitter tip. The effective diameter of the tip extracted from the Fowler-Nordheim plot[11] based on the $\mathrm{I}-\mathrm{V}$ curve was found to be around $12 \mathrm{~nm}$.

The e-beam currents at the first electron lens were 200 $\mathrm{nA}$ at the bias voltage of $-300 \mathrm{~V}$, and increased up to 800 $\mathrm{nA}$ at $-350 \mathrm{~V}$. The electron current at the sample at -300
$\mathrm{V}$ bias was measured to be $0.25 \mathrm{nA}$, which meant that more than $0.1 \%$ of the emitted electrons reached the sample.

The Einzel lens was used to focus the electron beam by applying a retarding or an accelerating bias to the center electrode of the Einzel lens. The other two electrodes of the Einzel lens are usually grounded, and hence the energy of the electron beam arriving at the sample depends only on the potential applied to the emitter tip. The focused electrons coming from the Einzel lens can be used for lithography or for other applications like imaging.

To evaluate the performance of the microcolumn, the images of a $\mathrm{Cu}$ grid sample were captured for both retarding and accelerating modes. Fig. 5(b) shows the result. For the retarding mode, the grid pattern was distinguishable when the bias voltage applied to the Einzel lens was lower than $-200 \mathrm{~V}$ and the clearest image was obtained at the bias voltage of $-253 \mathrm{~V}$. For accelerating mode, the biases for the distinguishable and clearest image were $+850 \mathrm{~V}$ and +934 V, respectively. While the accelerating mode shows better resolution, which is expected theoretically, it requires higher voltage bias for concentration of the e-beam. Since the insulation layer between the electrodes is extremely thin in the microcolumn, the retarding-mode operation is preferred for long-time stability. The result proved that the electric current arriving at the sample was sufficiently large to use the microcolumn as an e-beam source. 


\section{CONCLUSION}

We have developed an efficient e-beam source, a microcolumn, that can be used as a source module which covers $\mathrm{THz}$ and far infrared regions of the EM spectra. To achieve the low operating voltage, we developed a very sharp emitter tip by electrochemically etching a tungsten wire. The effective diameter of the tip estimated by Fowler-Nordheim plot was about $12 \mathrm{~nm}$. To obtain a large current at the end of the microcolumn, high-quality electron lenses were fabricated and aligned very precisely by observing the laser diffraction patterns. More than $0.1 \%$ of the electrons emitted by the tip passed through the microcolumn, which made it possible to capture the SEM image of a $\mathrm{Cu}$ grid sample (mesh 1000) and confirmed the potential of the microcolumn as an electron source module.

\section{ACKNOWLEDGMENT}

This research was supported by Basic Science Research Program through the National Research Foundation of Korea (NRF) funded by the Ministry of Education, Science and Technology (2009-0033).

\section{REFERENCES}

1. S.-S. Choi, J. S. Kim, E. D. Fabrizio, M. Gentili, H. B. Chung, H. J. Yoo, and B. W. Kim, "A novel X-ray mask for mix-and-match of optical and X-ray lithography applied in SOI device fabrication,” J. Korean Phys. Soc. 32, 727-730 (1998).

2. S. Kwon, P. Kim, S. Jeong, W. Chang, C. Chun, and D.-Y, Kim, "Fabrication of nano dot and line arrays using NSOM lithography,” J. Opt. Soc. Korea 9, 16-21 (2005).
3. D.-G. Kim, S.-Y. Woo, D.-K. Kim, T.-Y. Hwang, S.-H. Park, and D. Y. Kim, "Generation of 1.5 Gbps pseudorandom binary sequence optical signals by using a gain switched Fabry-Perot semiconductor laser," J. Opt. Soc. Korea 9, 103-106 (2005).

4. C. Kang, C.-S. Kee, I.-B. Sohn, and J. Lee, "Spectral properties of THz-periodic metallic structures," J. Opt. Soc. Korea 12, 196-199 (2008).

5. H. Jeong, J. Jeong, G. H. Song, and Y.-D. Jho, "Excitation wavelength dependence of terahertz radiation from InAs: UV versus IR,” J. Korean Phys. Soc. 56, 262-264 (2010).

6. N. E. Yu, C. Kang, H. K. Yoo, C. Jung, Y. L. Lee, C.-S. Kee, D.-K. Ko, and J. Lee, "Temperature dependent terahertz generation at periodically poled stoichiometric lithium tantalate crystal using femtosecond laser pulses," J. Opt. Soc. Korea 12, 200-204 (2008).

7. M. L. Yu, B. W. Hussey, H.-S. Kim, and T. H. P. Chang, "Emission characteristics of ultrasharp cold field emitters," J. Vac. Sci. Technol. B 12, 3431-3435 (1994).

8. H. S. Kim, M. L. Yu, E. Kratschmer, B. W. Hussey, M. G. R. Thomson, and T. H. P. Chang, "Miniature Schottky electron source," J. Vac. Sci. Technol. B 13, 2468-2472 (1995).

9. E. Kratschmer, H. S. Kim, M. G. R. Thomson, K. Y. Lee, S. A. Rishton, M. L. Yu, S. Zolgharnain, B. W. Hussey, and T. H. P. Chang, "Experimental evaluation of a $20 \times 20$ mm footprint microcolumn," J. Vac. Sci. Technol. B 14, 3792-3796 (1996).

10. S. Ahn, D. W. Kim, H. S. Kim, C. G. Park, and S. J. Ahn, "Study on the precise alignment of micro-lenses in the microcolumn by using the laser diffraction pattern," Mat. Sci. Forum 544-545, 845-848 (2007).

11. M. L. Yu, H.-S. Kim, B. W. Hussey, T. H. P. Chang, and W. A. Mackie, "Energy distributions of field emitted electrons from carbide tips and tungsten tips with diamondlike carbon coatings," J. Vac. Sci. Technol. B 14, 3797-3801 (1996). 